\title{
Novel Method for Precision Controlled Heating of TEM Thin Sections to Study Reaction Processes
}

\author{
Kinga A. Unocic, ${ }^{1}$ Lawrence F. Allard, ${ }^{1}$ Dorothy W. Coffey, ${ }^{1}$ Karren L. More ${ }^{2}$ \\ and Raymond R. Unocic ${ }^{2}$ \\ ${ }^{1 .}$ Materials Science \& Technology Division, Oak Ridge National Laboratory, Oak Ridge, TN, USA \\ ${ }^{2}$ Center for Nanophase Materials Sciences, Oak Ridge National Laboratory, Oak Ridge, TN, USA
}

Improving the efficiency of gas turbine engines necessitates the development of structural alloys that can operate at higher temperatures. The alloys must maintain structural integrity while possessing excellent high-temperature oxidation resistance. The formation of a stable oxide scale is needed to protect the material from aggressive oxidizing environments [1]. Previous research has shown that the addition of reactive elements (RE), such as $\mathrm{Hf}, \mathrm{Y}, \mathrm{La}$, Ti, improves oxidation resistance of the materials by improving oxide-scale adhesion and decreasing the overall oxide growth rate [1-3]. Oxide-scale growth is retarded by the segregation of RE elements to oxide grain boundaries, which effectively block cation diffusion from the underlying base material. Often, the structure and chemistry of the oxide scale is characterized following exposure to high-temperature oxidation [3]. Direct heating of TEM thin-foil specimens in an oxidizing environment is a useful method to study oxide growth kinetics. The recent development of a heating capability based on MEMS-fabricated heater devices that are used for in situ microscopy studies offers the potential to study thin alloy specimens, with precision control of temperature at heating and cooling rates up to $10^{6}{ }^{\circ} \mathrm{C} / \mathrm{s}$ [4]. With proper specimen preparation, devices such as Protochips Aduro ${ }^{\mathrm{TM}}$ E-chip heaters offer the capability to stop testing at any time to examine oxide scale chemistry and elemental segregation with electron energy-loss spectroscopy (EELS) or energy dispersive X-ray spectroscopy (EDS).

In the present work, we seek to characterize the high-temperature oxidation behavior of a Ti/Hf-doped $\mathrm{NiAl}$ alloy. Two different sample preparation approaches were used to prepare the alloy so that electrontransparent specimens could be attached to a Protochips Aduro heater chip. The first method used focused ion beam (FIB) milling to prepare thin TEM cross sections, as shown in Fig. 1a. The TEM lamellum was further Ar-ion nanomilled locally to create multiple electron-transparent tips. The specimen was then attached in the FIB instrument to the E-chip heater membrane with tungsten (Fig. 1a). The second process used an electropolishing method similar to preparing needle-like specimens for the atom probe [5]. Following electropolishing, the NiAl tip was FIB-sectioned (Fig. 1b) and attached to the heater membrane with tungsten. It is important to minimize Ga implantation during the sample preparation procedure. FIB milling resulted in Ga enrichment on the surface of the TEM specimen, while the electropolished specimen showed virtually no Ga present in the region of interest (Fig. 1e). The shapes of the tips, prepared using both methods, were kept similar in order to better compare the results of the heating experiments (Fig. 1c,d). In an initial heating experiment, a similar electropolished sample was heated in the column vacuum at $1000^{\circ} \mathrm{C}$, with a heating ramp of $10^{\circ} \mathrm{C} / \mathrm{s}$ to minimize the chance for specimen detachment from the heater due to differential thermal expansion. The electropolished tip was initially clean, as shown in the BF-TEM image of Fig. $2 \mathrm{a}$, but after $40 \mathrm{~s}$ heating at $1000^{\circ} \mathrm{C}$, some particles began to appear at the surface (Fig. 2b), and after $60 \mathrm{~s}$ total heating time, the surface showed even further development of a particle layer (Fig. 2c). Subsequent EDS analysis of the tip showed that the particles were Ti-rich (Fig. 2d), and were likely Ti oxide, from the residual $\mathrm{O}_{2}$ in the column. This work demonstrates the potential for in situ heating using a MEMS-based technology to study oxidation mechanisms in high-temperature structural alloys [6]. 


\section{References:}

[1] B.A. Pint, Oxidation of Metals, 45 (1996) p. 1.

[2] P.Y. Hou and K. Priimak, Oxidation of Metals, 63 (2005), p. 113.

[3] K.A. Unocic et al., Surface Coating and Technology. 6 (2007), p. 241.

[4] L.F. Allard, et al., Microscopy Research \& Technique 72(3) (2003), p. 208.

[5] M.K. Miller and G.D.W. Smith, Atom Probe Microanalysis: Principles and Applications to Materials Problems, (Materials Research Society, Pittsburgh, PA) (1989) pp. 37-59

[6] Research supported by ORNL's Center for Nanophase Materials Sciences, which is sponsored by the Scientific User Facilities Division, Office of Basic Energy Sciences, U.S. Dept. of Energy.
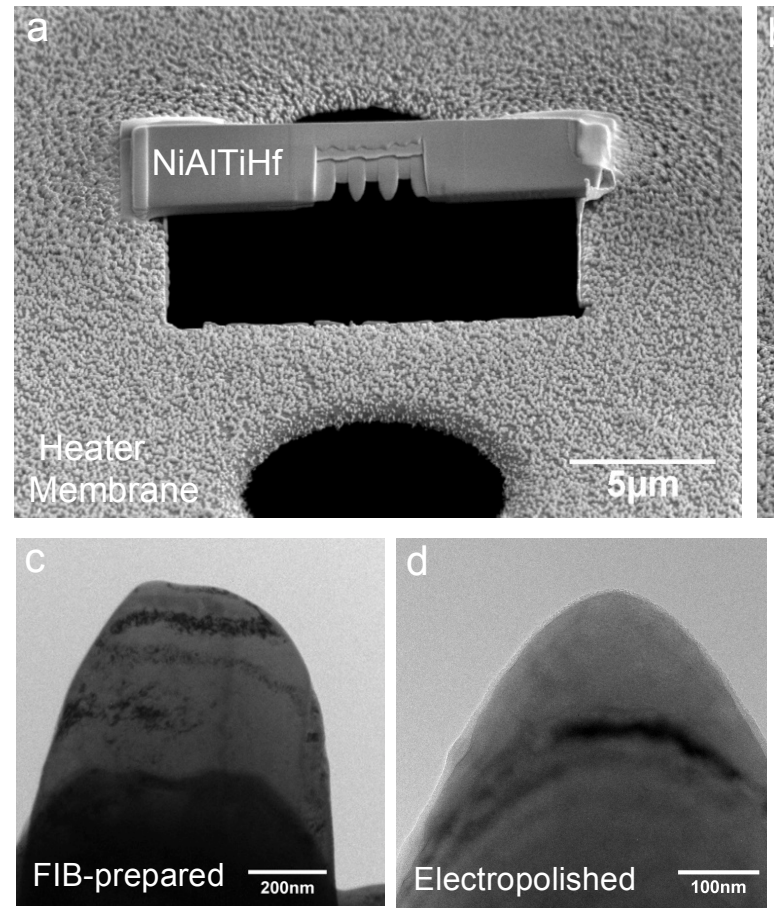
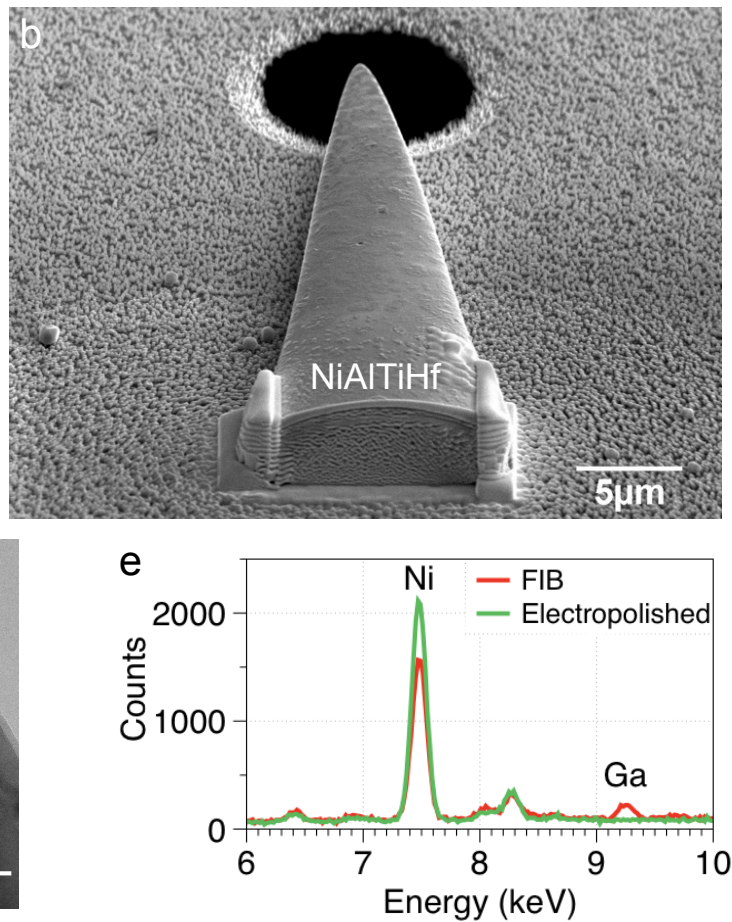

Figure 1. SE image of Ti/Hf-doped NiAl alloy prepared by a) FIB milling and b) electropolishing+FIB milling before heating experiments in vacuum. BF-TEM images showing tips prepared by c) FIB milling
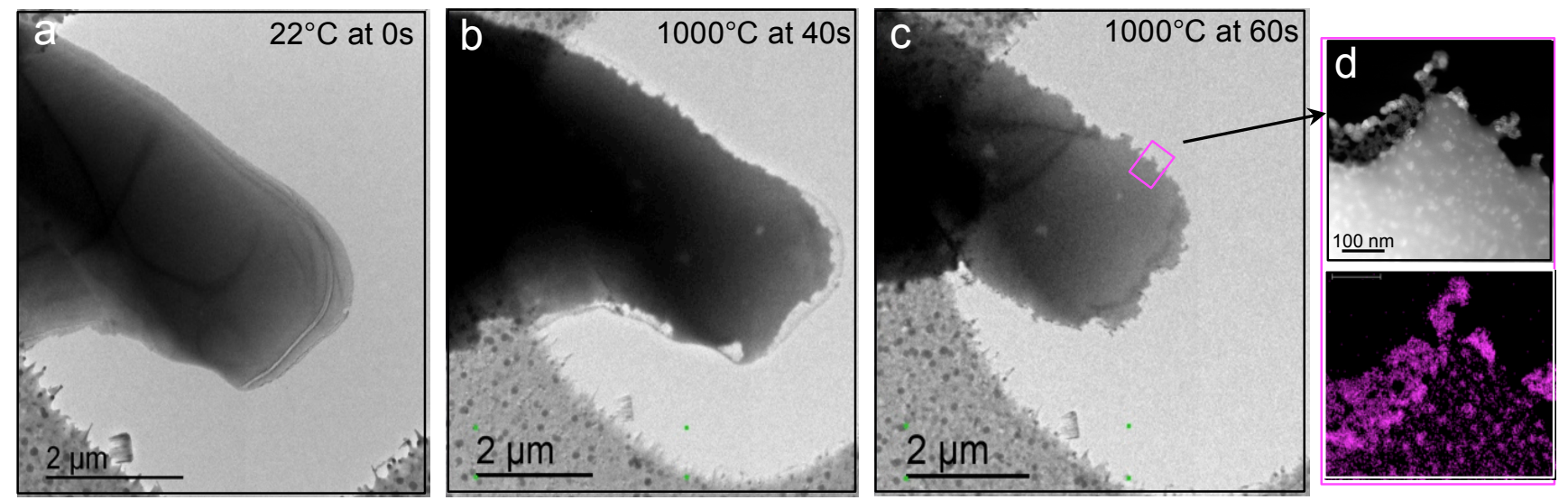

Figure 2. BF-TEM images of the electropolished tip a) before heating, b) during heating after 40s at $1000^{\circ} \mathrm{C}$, c) after $60 \mathrm{~s}$ at $1000^{\circ} \mathrm{C}$, and d) HAADF-STEM image of edge of tip (inset) with Ti EDS map following high-temperature exposure. 